\title{
Síndrome de Sengers: comunicación de dos casos en Chile.
}

\author{
Nathan Pulido ${ }^{1}$, Julio Rivera ${ }^{2}$, Marta Arriaza $^{3}$, Miguel Saavedra ${ }^{4}$, Gustavo Ríos ${ }^{5}$.
}

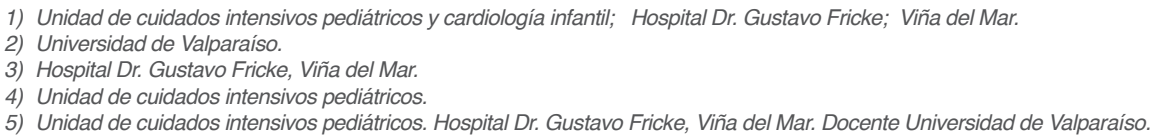

Resumen: El Síndrome de Sengers es una enfermedad mitocondrial autosómica recesiva, producida por mutación del gen de la Acil-Glicerol Kinasa (AGK), recientemente descubierto. Se caracteriza por cataratas congénitas bilaterales, miocardiopatía hipertrófica y acidosis láctica. Puede tener miopatía esquelética leve, intolerancia al ejercicio y desarrollo mental normal. Los pacientes fallecen tempranamente debido a falla cardíaca.

Dada la alta letalidad, lo infrecuente de este síndrome y la presencia de un diagnóstico confirmado, se presenta el caso clínico de 2 hermanos chilenos, fallecidos por la enfermedad, que se presentaron con el cuadro característico de cataratas congénitas bilaterales, miocardiopatía hipertrófica y acidosis láctica. El mayor, se operó las cataratas a los 4 meses de edad y falleció a la edad de 13 meses debido a falla cardíaca severa refractaria y falla orgánica múltiple, descompensado por una infección respiratoria. El menor se diagnosticó a los 3 meses de edad y se le confirmó la mutación del gen de AGK en Alemania. Se decidió no operarlo de las cataratas dado el mal pronóstico vital. Presentó progresión de la miocardiopatía hipertrófica y falleció súbitamente a los 8 meses de edad. 


\section{Sengers Syndrome: report of two cases in Chile.}

Senger's Syndrome is a recessive autosomal mitochondrial disease due to a recently discovered mutation of the Acyl-Glycerol Kinase (AGK) gen,. It is characterized by congenital bilateral cataracts, progressive hypertrophic cardiomyopathy and lactic acidosis. It may present skeletal myopathy, exercise intolerance and usually normal mental development. Patients die early in life due to heart failure.

The clinical cases of two brothers with a confirmed diagnosis of Senger's syndrome are reported. The older brother was operated on for cataracts at

\section{Introducción:}

El Síndrome de Sengers, descrito en 1975, se caracteriza por cataratas congénitas bilaterales, miocardiopatía hipertrófica progresiva y acidosis láctica desencadenada por el esfuerzo físico. Generalmente tiene desarrollo mental normal. Puede presentarse en una forma neonatal severa con muerte temprana y una forma tardía con sobrevida hasta la cuarta década. Se caracteriza por alteraciones de las mitocondrias, con depósito citoplasmático de lípidos y glicerol en músculo cardíaco y esquelético. Es muy infrecuente, con alrededor de 40 casos descritos, principalmente en Europa ${ }^{1-9}$.

Es una Enfermedad Mitocondrial debida a una mutación del gen de la Acil-Glicerol Kinasa (AGK), con herencia autosómica recesiva, recientemente descubierto $(2012)^{10}$ presenta el caso clínico de 2 hermanos chilenos fallecidos por Síndrome de Sengers, en los cuales se pudo confirmar el diagnóstico, mediante la identificación de la mutación del gen de la AGK.

\section{Casos Clínicos:}

Se trata de 2 hermanos que se presentaron simultáneamente, al sufrir una infección respiratoria durante el invierno de 2012.

\section{Caso Clínico 1.}

Recién nacido de término, con peso adecuado (3080 Gr) para la edad gestacional (39 semanas). Nació con the age of 4 months and he died when he was 13 months old due to severe refractory heart failure and multi-organ failure, decompensated by a respiratory infection. The younger brother was diagnosed at 3 month of age and the AGK gene mutation was confirmed in Germany. Cataracts were not operated on due to the the patient's extremely poor prognosis. He had progressive hypertrophic cardiomyopathy and died suddenly at 8 month of age. Key Words: Sengers Syndrome, Hypertrophic Cardiomyopathy, Acyl-Glycerol-Kinase, Mitochondrial Disease, Congenital Cataract, Lactic Acidosis.

Apgar 9-9 por cesárea electiva y su madre tenía Púrpura Trombocitopénico Idiopático (PTI). Los padres no eran consanguíneos. El recién nacido presentó Síndrome de Dificultad Respiratoria, Neumonía y Sepsis de origen ocular. Se planteó posible cardiopatía congénita por cardiomegalia radiológica y se trasladó a la Unidad de Neonatología de nuestro hospital. Evolucionó con trombocitopenia severa secundaria a PTI materno, con recuentos de 7.000-9.000 plaquetas, que se recuperó gradualmente. Presentó acidosis metabólica leve (pH: ${ }^{7,38}$, Bicarbonato: 14 y Exceso de Base (BE): -9) con Ácido láctico elevado (39,7mg\%) (VN: 6-19 mg). El Ecocardiograma Doppler Color fue normal, descartándose miocardiopatías y cardiopatías congénitas. Evolucionó satisfactoriamente con tratamiento antibiótico. Se planteó descartar una enfermedad metabólica en control ambulatorio.

Se operó de cataratas congénitas bilaterales, a la edad de 3 meses, sin incidentes.

A los 13 meses de edad presentó cuadro respiratorio febril, de 3-4 días de evolución, de coriza, tos y fiebre, agravándose rápidamente. Ingresó a la Unidad de Cuidados Intensivos Pediátricos con diagnósticos de Bronquiolitis y Neumonía. Evolucionó con shock, insuficiencia renal y respiratoria, acidosis metabólica severa $(\mathrm{pH}$ : 6,7-6,8; BE: -26 a -29; Bicarbonato: ${ }^{7-8}$. Se conectó a ventilación mecánica, se inició Dopamina y Dobutamina. Se agregó antibióticos por sospecha de infección bacteriana. La radiografía mostró cardiomegalia e infiltrados algodonosos 
bilaterales. Sangró por sitios de punción, con Protrombina de $11 \%$, TTPA incoagulable y Plaquetas de 122.000 . Presentó hepatomegalia con transaminasas elevadas (GOT: 388-513, GPT: 141). Troponina ${ }^{2,17}$ y CPK totales (327) y MB (134) elevadas. Lactato elevado (58mg\%)(VN: 6-19 mg\%). Se inició Hemofiltración Veno-Venosa Continua (HFVVC) por falla renal. Al tercer día fue evaluado por cardiología infantil, en shock carcinogénico refractario a 3 inótropos y falla orgánica múltiple, en ventilación mecánica y HFVVC. La ecocardiografía mostró miocardiopatía hipertrófica concéntrica, con grosor de septum y pared posterior del ventrículo izquierdo de $13 \mathrm{~mm}(\mathrm{VN}$ : $5 \mathrm{~mm}$ ), con aspecto infiltrativo; sin obstrucción del tracto de salida del ventrículo y contractilidad muy disminuida. No había derrame pericárdico, anomalías del origen de las arterias coronarias ni malformaciones cardíacas congénitas. Con el antecedente de cataratas congénitas operadas y de hermano menor con cataratas congénitas no operadas, se planteó el diagnóstico de una probable enfermedad metabólica o mitocondrial. El cuadro clínico concordaba con Síndrome de Sengers. Evolucionó con falla multi-orgánica severa, refractaria al manejo intensivo y acidosis metabólica que no respondió a bicarbonato. Falleció al $5^{\circ}$ día de evolución.

En su autopsia el estudio macroscópico demostró hipertrofia concéntrica del ventrículo izquierdo, con grosor de 13-14 mm. El corazón pesaba 135 grs. (VN: 40grs). La microscopia óptica demostró áreas de hipertrofia, con vacuolización de las miofibrillas cardíacas, acentuada granulación citoplasmática y pérdida de las estrías del tejido muscular esquelético, compatible con Síndrome de Sengers (Figuras 1 y 2). No se efectúó microscopia electrónica en nuestro hospital.

\section{Caso Clínico 2.}

Lactante de 1 mes 23 días de edad, con historia de 3 días de coriza, tos y dificultad respiratoria. Se trató en forma ambulatoria con agonistas beta ${ }^{2}$ inhalados y kinesioterapia, por cuadro bronquial obstructivo. Ingresó al mismo tiempo que su hermano (caso clínico 1). La radiografía mostró condensación del lóbulo superior derecho y retro cardíaca. La inmunofluorescencia indirecta (IFI) fue positiva para Virus Sincicial Respiratorio (VRS). La PCR estaba en rango de infección no bacteriana. Se ingresó con diagnóstico de Bronquiolitis y Neumonía por VRS. Se manejó con oxígeno y kinesioterapia. Se confirmó el diagnóstico previo de cataratas bilaterales. Se efectuó ecocardiograma que reveló Miocardiopatía Hipertrófica, con aspecto heterogéneo tipo infiltrativo, con septum y pared posterior del VI con grosor de 7-8 $\mathrm{mm}$ (VN: $4 \mathrm{~mm}$ ), sin obstrucción del tracto de salida. La radiografía no mostró cardiomegalia. Con estos hallazgos se confirmó el diagnóstico (familiar) de Síndrome de Sengers. Presentó empeoramiento del cuadro respiratorio y mayor requerimiento de oxígeno, por lo que se inició antibióticos. El lactato estuvo elevado, entre 34 y $189 \mathrm{mg} \%$ (VN: 6-19). Amonio normal (38 ug\%)(VN: 29-70 ug\%). Presentó acidosis metabólica (pH: 7,21; Bicarbonato: 16; BE: -7) leve y transitoria. Se solicitó Tándem y se inició Carnitina oral. El screening metabólico mostró: Cloruro Férrico: (-). DNF: (-). Benedict: (+). Aminoacidemia normal y aminoaciduria alterada (glicina, serina, glutamina). Se recuperó lentamente del cuadro respiratorio. Las radiografías de control mostraron cardiomegalia. Presentó una infección intrahospitalaria por Rotavirus, que requirió de fleboclisis, con sobre-hidratación secundaria que se trató con restricción hídrica y furosemida. Se dio de alta,

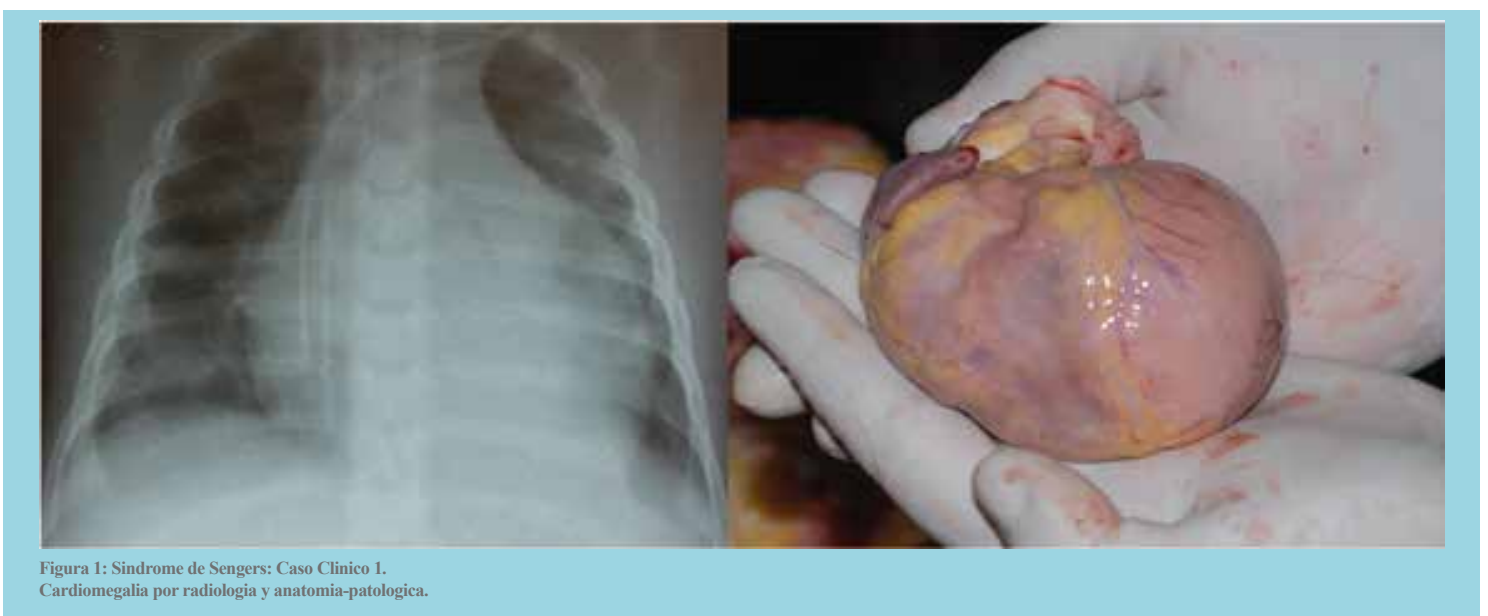




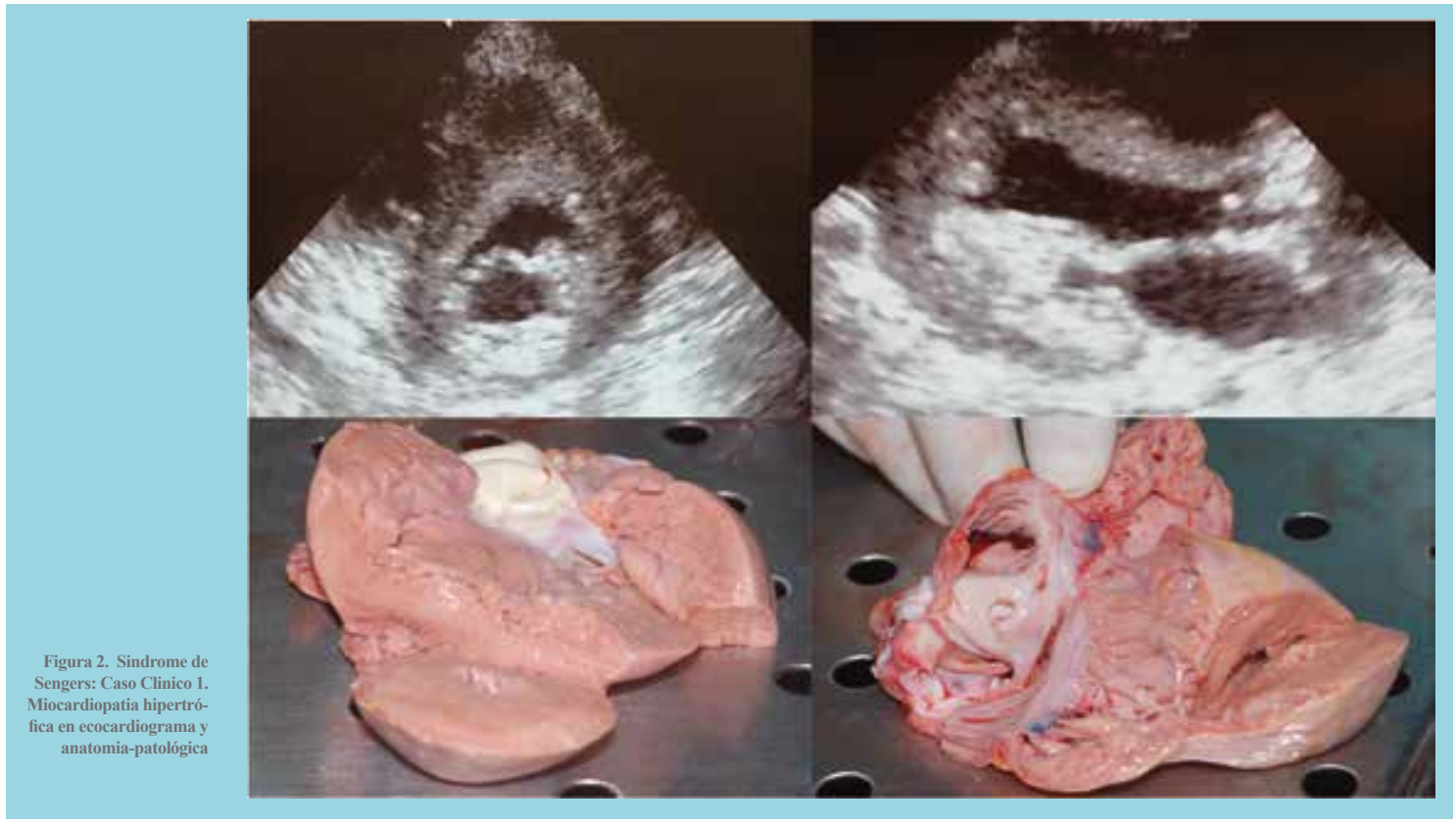

luego de 3 semanas. La evaluación neurológica confirmó un Síndrome Hipotónico leve, con movimientos y reflejos normales.

Se confirmó el diagnóstico de Síndrome de Sengers, mediante el estudio del gen de la Acil-Glicerol Kinasa (AGK). Se encontró una mutación heterocigota compuesta del gen AGK: c.871C>T p.Q291*, c.1035insT p.1346Yfs. (Múnich, Alemania).

Se controló ambulatoriamente en cardiología. El paciente se mantenía sin signos de falla cardíaca ni dificultad respiratoria, sin soplo, galope ni arritmias. La ecocardiografía evidenció un aumento del grosor del septum y pared posterior del VI (10 y $12 \mathrm{~mm})$, con buena contractilidad y sin obstrucción del tracto de salida del VI. El electrocardiograma mostraba signos de hipertrofia ventricular izquierda. Se mantuvo con carnitina oral y se suspendió la furosemida. En conjunto con la familia, se decidió no operar las cataratas congénitas bilaterales, dado el alto riesgo anestésico y mal pronóstico vital. Se controló en neurología, encontrándose un retraso leve del desarrollo psicomotor y vagabundeo ocular por las cataratas congénitas (Figuras 3 y 4 ).

Falleció súbitamente a la edad de 8 meses, en relación a un cuadro respiratorio alto leve. No se efectuó autopsia por tener confirmado el diagnostico de Síndrome de Sengers.

\section{Discusión:}

Los 2 pacientes descritos presentaron las características clínicas típicas del Síndrome de Sengers, consistentes en cataratas congénitas bilaterales, miocardiopatía hipertrófica progresiva y acidosis láctica. Este síndrome fue descrito en 1975 por RCA Sengers y colaboradores. Al igual que en otras series de casos, no se encontró un retraso importante del desarrollo mental, ni hipotonía muscu$\operatorname{lar}^{1-8}$. Ambos hermanos presentaron descompensación metabólica y cardíaca grave en relación a infecciones respiratorias intercurrentes, con desenlace fatal. Por esto, se planteó el diagnóstico de Síndrome de Sengers o alguna otra enfermedad mitocondrial.

Este síndrome se puede presentar en una forma neonatal grave y otra más leve, con sobrevida hasta la cuarta década. Las características clínicas, bioquímicas y anátomopatológicas son similares ${ }^{1-8}$. En general, se produce leve compromiso mental y miopático esquelético. Sólo un estudio en recién nacidos, con la forma grave, demostró hipoplasia de cerebelo, tronco cerebral y de hemisferios cerebrales e infartos corticales ${ }^{9}$. Estos pacientes presentaban miocardiopatía dilatada y no hipertrófica, no tenían confirmación genética, por lo que el diagnóstico no es seguro.

La miocardiopatía hipertrófica puede ser idiopática o secundaria a otras causas, como las enfermedades mitocondriales y metabólicas, especialmente cuando se 

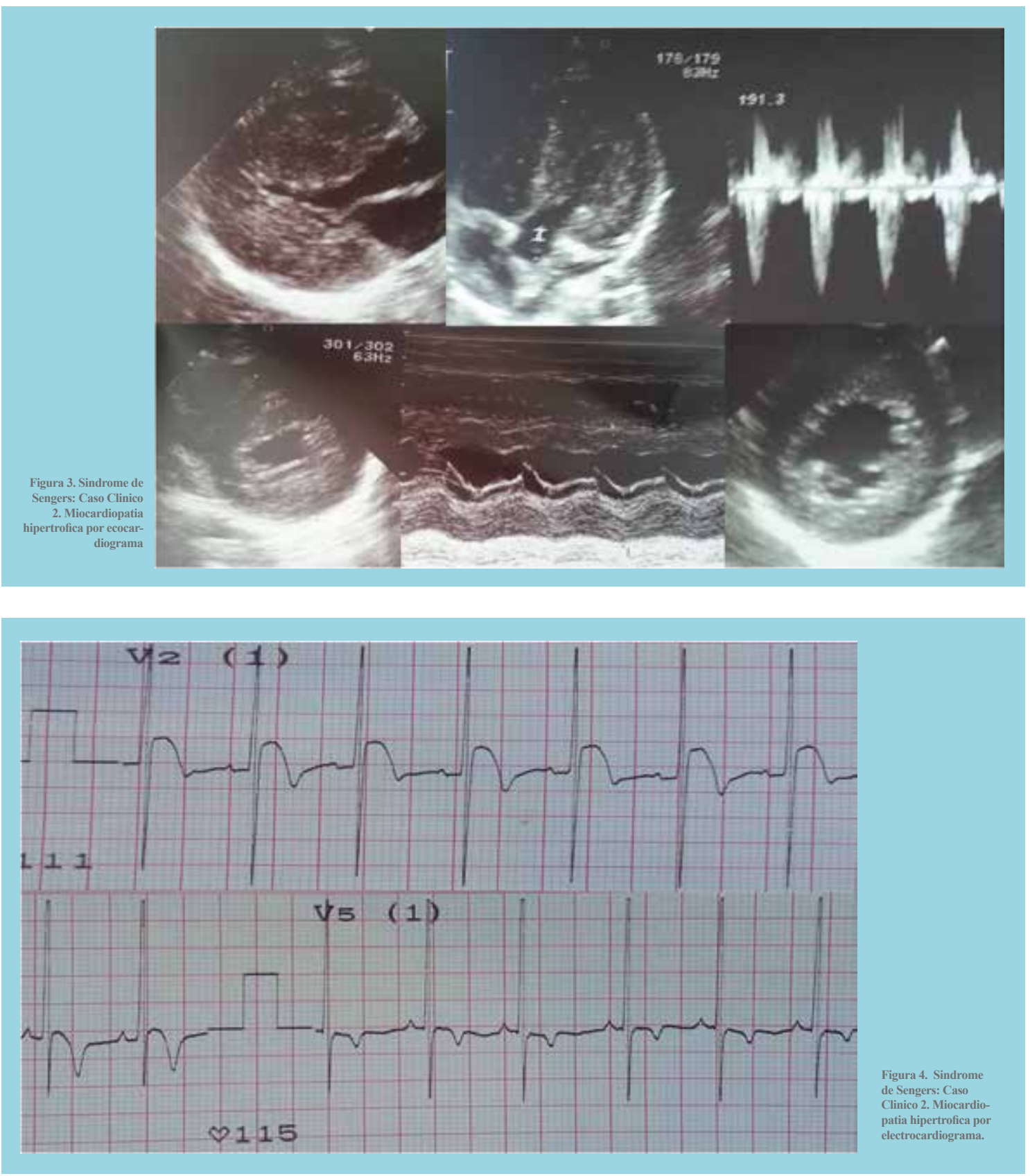

asocia miopatía esquelética y cardíaca, en presencia de acidosis metabólica y/o láctica. En muchos casos no es posible avanzar en la etiología exacta de una enfermedad metabólica o mitocondrial, por no contar con laboratorios especializados en genética molecular o microscopia electrónica. Algunas enfermedades mitocondriales se deben a mutaciones o deleciones del DNA Mitocondrial. Otras son producidas por depleción del DNA Mitocondrial, se- cundario a mutaciones del DNA Nuclear, responsable de la mantención de la integridad o de la síntesis del DNA Mitocondrial. Pueden tener herencia materna, esporádica o mendeliana (recesivas o dominantes) ${ }^{11}$.

Recientemente (Febrero 2012), se encontró la causa genética de la mayoría de los casos de Síndrome de Sengers. Se trata de la mutación del gen de una proteína mitocondrial, la Acil-Glicerol Kinasa (AGK), 
que al faltar produce alteraciones del metabolismo de lípidos, glicerol y nucleótidos. La AGK es una kinasa que fosforila Di-Acil Glicerol y Mono-Acil Glicerol en Ácido Fosfatídico y Liso-Fosfatídico, precursores de la Cardiolipina y otros fosfolípidos. La falla de AGK es responsable de alteración del ensamblaje de "adenine nucleotide translocator 1" (ANT1) en la membrana interna de la mitocondria. El defecto de ANT1 es una de las causas de alteración de la síntesis de ATP, junto a las alteraciones de la ATP Sintetasa y el Transportador de Fosfato Mitocondrial. Esto es importante en las células musculares cardíacas y esqueléticas, por ser dependientes del metabolismo de lípidos y no de glucosa ${ }^{10,11}$. La ANT1 es una de las enzimas que participa activamente en la mantención del pool de ATP para la maquinaria productora de energía de la mitocondria. Cuando esto falla se produce depleción del DNA mitocondrial, alteración de la producción energética y alteración del metabolismo de ácidos grasos y glicerol. En el Síndrome de Sengers se describió una deficiencia severa del ANT1, sin encontrar su causa genética especifica mediante análisis mutacional y de linkage. Se planteó la hipótesis de una alteración a nivel de la transcripción, traducción o post-traducción ${ }^{12}$. En un caso de Síndrome de Sengers se encontró una mutación del gen de ANT $1^{13}$. Se ha desarrollado ratones deficientes en ANT1, con Síndrome de Sengers ${ }^{14}$. En casos de cataratas familiares, se encontró una mutación del gen de AGK, con cataratas, acidosis láctica, miocardiopatía y accidentes vasculares cerebrales ${ }^{15}$. En pacientes con Síndrome de Sengers, por mutación de AGK, se ha observado cristales de Citrato Sintetasa en las mitocondrias. Algunos pacientes tenían accidentesvasculares cerebrales16. El Síndrome de Sengers comparte características clínicas con otras enfermedades mitocondriales: el Síndrome MELAS, con encéfalo-miopatía, acidosis láctica y accidentes vasculares cerebrales, producido por mutaciones del DNA Mitocondrial ${ }^{17}$ y el Síndrome de Barth que se caracteriza por miopatía, neutropenia y miocardiopatía debido a una mutación del gen de la Tafazzina (TAZ), necesaria para la producción de Cardiolipina ${ }^{18}$. La mutación del gen del transportador mitocondrial de fosfato produce una alteración de la fosforilación oxidativa, con acidosis láctica, miocardiopatía hipertrófica e hipotonía muscular ${ }^{19,20}$.

\section{Referencias:}

1. SENGERS RCA, TER HAAR BGA, TRIJBELS JMF, WILLEMS JH, DANIELS G, STADHOUDERS AM. Congenital cataracts and mitochondrial myopathy of skeletal and heart muscle associated with lactic acidosis after exercise. J Pediatr 1975; 86:873-80.

2. SENGERS RC, STADHOUDERS AM, VAN LAKWIJK-VONDROVICOVA E, KUBAT K, RUITENBEEK W. Hypertrophic cardiomyopathy associated with a mitochondrial myopathy of voluntary muscles and congenital cataract. Br Heart J. 1985; 54:543-7.
3. CRUYSBERG JR, SENGERS RC, PINCKERS A, KUBAT K, VAN HAELST UJ. Features of a syndrome with congenital cataract and hypertrophic cardiomyopathy. Am J Ophtalmol. 1986; 102:740-9.

4. VAN EKEREN GJ, STADHOUDERS AM, EGBERINK GJ, DANIELS O, KUBAT K. Hereditary mitochondrial hypertrophic cardiomyopathy with mitochondrial myopathy of skeletal muscle, congenital cataract and lactic acidosis. Virchows Arch A Pathol Anat Histopathol. 1987; 412:4752.

5. SMEITINK JA, SENGERS RC, TRIJBELS JM, RUITENBEEK W, DANIELS O, STADHOUDERS AM, et 
al. Fatal neonatal cardiomyopathy associated with cataract and mitochondrial myopathy. Eur J Pediatr. 1989; 148:656-9.

6. VAN EKEREN GJ, STADHOUDERS AM, SMEITINK JA, SENGERS RC. A retrospective study of patients with the hereditary syndrome of congenital cataract, mitochomdrial myopathy of heart and skeletal muscle and lactic acidosis. Eur J Pediatr. 1993; 152:255-9.

7. LIAO SL, HUANG SF, LIN JL, LAI SH, CHOU YH, KUO CY. Syndrome of mitochondrial myopathy of heart and skeletal muscle, congenital cataract and lactic acidosis. Acta Pediatr Taiwan. 2003; 44:360-4.

8. ATIQ M, IQBAL S, IBRAHIM S. Sengers disease: a rare association of hypertrophic cardiomyopathy and congenital cataracts. Indian J Pediatr. 2004; 71:437-40.

9. PERRY MS, SLADKY JT. Neuroradiologic findings in Sengers syndrome. Pediatr Neurol. 2008; 39:113-5.

10. MAYR JA, HAACK TB, GRAF E, ZIMMERMANN F, WIELAND T, HABERBERGER B, et al. Lack of the mitochondrial protein Acylglycerol Kinase causes Sengers syndrome. The American Journal of Human Genetics. 2012; 90:314-20.

11. HUANG CC, HSU CH. Mitochondrial disease and mitochondrial DNA depletion syndromes. Acta Neurol Taiwan. 2009; 18: 287-95.

12. JORDENS EZ, PALMIERI L, HUIZING M, VAN DEN HEUVEL LP, SENGERS RC, DORNER A, et al. Adenine Nucleotide Translocator 1 deficiency associated with Sengers syndrome. Ann Neurol. 2002; 52:95-99.

13. ECHANIZ-LAGUNA A, CHASSAGNE M, CERESUELA J, ROUVET I, PADET S, ACQUAVIVA C, et al. Complete loss of expression of the ANT1 gene causing cardiomyopathy and myopathy. J Med Genet. 2012;
49:146-50.

14. GRAHAM BH, WAYMIRE KG, COTTRELL B, TROUNCE IA, MAC GREGOR GR, WALLACE DC. A mouse model for mitochondrial myopathy and cardiomyopathy resulting from a deficiency in the heart/muscle isoform of the adenine nucleotide translocator. Nat genet. 1997; 16: 22634 .

15. ALDAMESH MA, KHAN AO, MOHAMED YJ, ALGHAMDI MH, ALKURAYA FS. Identification of a truncation mutation of acylglycerol kinasa (AGK) gene in a novel autosomal recessive cataract locus. Hum Mutat. $2012 ; 33$ : 960-2.

16. SIRWARDENA K, MACKAY N, LEVANDOVSKIY V, RAIMAN J, KANTOR PF, ACKERLEY C, et al. Mitochondrial citrate synthase crystals: Novel finding in Sengers syndrome caused by acylglycerol kinasa (AGK) mutations. Mol Genet Metab. 2013; 108: 4050.

17. SCAGLIA F, NORTHROP JL. The mitochondrial myopathy encephalopathy, lactic acidosis with stroke-like episodes (MELAS) syndrome: a review of treatment options. CNS Drugs. 2006; 20: 443-64.

18. TAKEDA A, SUDO A, YAMADA H, IZUMI G, NISHINO I, ARIGA T. Eponym: Barth syndrome. Eur J Pediatr. 2011; 170: 1365-7.

19. MAYR JA, MERKEL O, KOHLWEIN SD, GEBHARDT BR, BHOLES H, FOTSCHL U, et al. Mitochondrial phosphate-carrier deficiency: a novel disorder of oxidative phosphorylation. Am J Hum Genet. 2007;80:478-84.

20. MAYR JA, ZIMMERMANN FA, HORVATH R, SCHEIDER HC, SCHOSER B, HOLINSKI-FEDER E, et al. Deficiency of the mitochondrial phosphate carrier presenting as myopathy and cardiomyopathy in a family with three affected children. Neurumuscul Disord. 2011; 21:803-8. 